\title{
Análise do Impacto das Publicações Científicas Qualificadas na Área de Ciências Agrárias I da Capes
}

\author{
Edilson Messias Gonçalves Júnior ${ }^{1 *}$, Eric Batista Ferreira ${ }^{2}$
}

\begin{abstract}
Resumo
No Brasil, alguns dos índices utilizados na avaliação da difusão da ciência são: o índice Qualis, os índices h, $g$ e $h$ normalizado além do fator de impacto e sua versão proprietária o JCR. Este artigo tem por objetivo avaliar o impacto e a qualidade dos periódicos da área de Ciências Agrárias I da Coordenação de Aperfeiçoamento de Pessoal de Nível Superior (CAPES), no período de 2010 a 2016. Essa avaliação se deu a partir de diversos vieses, sendo que para alguns puderam ser analisadas todas as 3975 revistas (censo) e, para outros, uma amostra de 748 revistas. Foram avaliados o Qualis, citações por artigo, número de artigos publicados, número de autores por artigo, índice g, índice h, índice $\mathrm{h}$ individual normalizado ( $\mathrm{hi}_{\text {norm }}$ ), número de citações, número de artigos, fator de impacto (FI) e JCR (Jornal Citation Reports). Os dados referentes ao Qualis foram obtidos da plataforma Sucupira da Capes, enquanto as demais variáveis foram coletadas a partir do software Harzing's Publish or Perish. O tratamento estatístico e a tabulação dos dados foram feitas no software estatístico R e no software Excel, respectivamente. Os resultados apontam que existe uma correlação positiva entre todas as métricas de impacto supramencionadas e uma correlação baixa do Qualis com as outras métricas de avaliação da produção científica.
\end{abstract}

Palavras-chave: Métricas. Correlação. Qualis. Indicadores.

\section{Impact Analysis of Qualified Scientific Publications in the Area of Agricultural Sciences I of Capes}

\begin{abstract}
In Brazil, some of the indices used to evaluate the diffusion of science are: the Qualis index, the normalized $\mathrm{h}$ index, $\mathrm{g}$ index and $\mathrm{h}$ index, in addition to the impact factor and its proprietary version the JCR. This article aims to evaluate the impact and quality of journals in the area of Agrarian Sciences I of the Coordination for the Improvement of Higher Education Personnel (CAPES), from 2010 to 2016. This evaluation was based on several biases, that for some, all 3975 magazines could be analyzed (census) and for others, a sample of 748 magazines. Qualis, citations per article, number of articles published, number of authors per article, $g$ index, $\mathrm{h}$ index, individual normalized $\mathrm{h}$ index (hinorm), number of citations, number of articles, impact factor (FI) and JCR (Journal Citation Reports) were evaluated. Qualis data were obtained from Capes Sucupira platform, while the other variables were collected using Harzing's Publish or Perish software. Statistical treatment and data tabulation were performed using R statistical software and Excel software, respectively. The results indicate that there is a positive correlation between all the above mentioned impact metrics and a low Qualis correlation with the other evaluation metrics of scientific production.
\end{abstract}

Keywords: Metrics. Correlation. Qualis. Indicators.

\footnotetext{
${ }^{1}$ Universidade Federal de Alfenas. Campus Alfenas-MG https://orcid.org/0000-0003-4295-6150

${ }^{2}$ Universidade Federal de Alfenas. Campus Alfenas-MG

https://orcid.org/0000-0003-3361-0908

*Autor para correspondência: edilsonmessiasjunior@gmail.com
}

Recebido para publicação em 05 de setembro de 2019. Aceito para publicação em 14 de outubro de 2019.

e-ISSN: 2447-6218 / ISSN: 2447-6218 / (C) 2009, Universidade Federal de Minas Gerais, Todos os direitos reservados. 


\section{Introdução}

A Cienciometria ou Cientometria é um ramo da sociologia das ciências que estuda as métricas de avaliação da produção científica de maneira geral, buscando correlações, nuances e vieses (Hayashi, 2012). Conhecer o impacto da ciência produzida no País, em particular na área de Ciências Agrárias é fundamental para subsidiar políticas de aporte de financiamento para pesquisa, traçar metas de aprimoramento da pesquisa e da pós-graduação nacionais e classificação do Brasil no cenário científico mundial. Além disto, devido ao grande volume de conhecimento científico produzido hoje em dia são necessárias métricas que possam reger e manter a qualidade do que é produzido no meio científico. Diante deste fato, procura-se fazer uma análise do impacto das produções científicas da área de Ciências Agrárias I da CAPES, com o intuito de responder quais as relações que permeiam todas estas métricas e como elas se correlacionam. A necessidade de se responder estas perguntas advém do fato de que, grande parte das pessoas que trabalham no meio científico não possuem conhecimento da forma como estas métricas são obtidas, e se sabem, se limitam ao índice Qualis. Existem estudos no Brasil que trazem um panorama da situação da produção científica nacional e do impacto que nossos artigos e periódicos possuem internacionalmente. Artigos similares a este são importantes para que todos os engajados com a ciência brasileira possam estar a par do funcionamento das métricas que regem a qualidade dos periódicos e artigos no país. A maioria destes trabalhos trazem a tona disparidade existente entre a produção nacional e internacional. De acordo com Packer (2010) em 2010 apenas 148 periódicos brasileiros estão indexados na plataforma Web of Science (WoS), caindo para 114 em 2015 como diz Rodrigues (2015). Ainda de acordo com Packer (2010) a área de Ciências Agrárias I, objeto de estudo deste artigo, não possuí resultado diferente, apenas $25 \%$ dos periódicos que publicam na área estão cadastrados no WoS.

Segundo Packer (2010), "o desempenho dos periódicos brasileiros nos rankings internacionais baseados em citações recebidas ainda é muito inferior aos dos periódicos dos países desenvolvidos, afetando o impacto da pesquisa brasileira como um todo." Tal fato parece ser justificado por Ferreira (2010) pois, segundo a autora a forma como os periódicos nacionais são avaliados geram muitas críticas internacionalmente.

De acordo com a CAPES, o índice Qualis avalia os programas de pós-graduação de forma indireta por meio dos periódicos no qual publicam seus professores. Indiretamente, o Qualis avalia a produção científica, porém não é seu objetivo principal. De acordo com Barata (2016), o Qualis faz uso de diferentes critérios de avaliação, um para cada área do conhecimento, o que justifica o fato de uma mesma revista possuir diferentes índices. Toda revista científica que foi citada pelo Programa em relatório Coleta CAPES, anualmente feito, recebe algum Qualis. A partir da chegada dessas informações, cada câmara utiliza seu próprio algoritmo para classificá-las e categorizá-las nos estratos A1, A2, B1, B2, B3, B4, B5 e C.

Segundo Hirsch (2005) "o índice h é uma métrica que pretende avaliar diretamente o impacto de um conjunto de publicações de um autor, de uma revista, uma universidade etc". Ele é calculado a partir da organização do número de citações que cada artigo científico possui em ordem decrescente. Isso feito, diz-se que o índice $h$ de um artigo é igual a h se os $h$ primeiros artigos de um periódico (ou de um autor) possuírem pelo menos $h$ citações.

De acordo com Harzing et al. (2014) "o índice hi norm (índice h normalizado) é o índice h para autores". Ainda de acordo com os autores, no cálculo deste índice normaliza-se o número de citações para cada artigo pela divisão do número de citações pelo número de autores deste artigo, então é calculado o índice h para estas citações normalizadas.

Já o índice g, segundo Egghe (2006) "é definido como o maior número $g$ de trabalhos que juntos receberam g ao quadrado citações". Também é necessário que você leitor entenda as diferenças e semelhanças de duas métricas estudadas neste artigo, o fator de impacto (FI) e o JCR.

De acordo com a Capes o JCR (Journal Citation Reports) é um recurso que permite avaliar e comparar publicações científicas utilizando dados de citações extraídos de revistas acadêmicas e técnicas e o impacto destas na comunidade científica. Pinto et al. (1999) dizem que o JCR é calculado levando-se em consideração os periódicos indexados na base de dados Science Citation Index $(S C I)^{3}$ da plataforma do Institute for Scientific Information (ISI).

Ainda de acordo com Pinto et al. (1999) "o JCR de uma revista é dado pela razão entre o número de citações contidas na plataforma do ISI nos últimos dois anos pelo número de artigos publicados neste mesmo intervalo de tempo". O FI é calculado desta mesma forma, porém, ele retira os dados dos periódicos do Google Escolar.

Diante das discussões apresentadas, objetiva-se neste artigo avaliar, de 2010 a 2016, indicadores de qualidade dos periódicos da área de Ciências Agrárias I da CAPES, que possuam Qualis no ano 2016. E tem por objetivos específicos analisar de 2010 a 2016 a evolução temporal do Qualis das revistas, do índice h, do índice $g$ e do JCR. 
Análise do Impacto das Publicações Científicas Qualificadas na Área de Ciências Agrárias I da Capes

\section{Material e métodos}

Por ser a principal linha de pesquisa dos autores, a área de Ciências Agrárias I foi selecionada para sofrer uma análise quantitativa e retrospectiva das métricas que avaliam sua produção científica. A pesquisa foi desenvolvida considerando-se dois intervalos de tempo, o triênio de 2010 a 2012 e o quadriênio de 2013-2016, períodos esses inspirados nos interstícios praticados pela CAPES. Para algumas variáveis, o estudo se deu a partir de um censo das 3975 revistas da área, e para outras, a partir de uma amostra de 748 revistas.. Foram analisadas métricas como: Qualis em cada área do conhecimento que dispunham classificação as revistas que apresentavam Qualis em Agrarias I, citações por artigo, número de artigos publicados, número de autores por artigo, índice $\mathrm{g}$, índice $\mathrm{h}$ e $\mathrm{h}$ individual normalizado ( $\mathrm{hi}$ norm ), número de citações, número de artigos, fator de impacto (FI), e JCR (Jornal Citation Reports). Com exceção do JCR, que foi coletado no portal Web of Knowledge, e do Qualis que foi coletado na plataforma Sucupira, as demais variáveis foram consultadas no software Harzing 's Publish or Perish (Harzing, 2007).

Também foi coletado o ISSN de cada de revista para evitar confusões com revistas homônimas. A tabulação dos dados e sua análise descritiva foram feitos pelo softwares Excel e R (R CORE TEAM, 2018) respectivamente. O coeficiente de correlação de Spearman (Siegel,; Castellan Jr, 2006) foi usado para calcular as correlações entre as variáveis, considerando-se sempre os dois intervalos de tempo. Para a obtenção do Qualis médio de cada área do conhecimento foi feita uma transformação da variável categórica Qualis em postos, a saber: $0=$ Não possui, $1=\mathrm{C}, 2=\mathrm{B} 5,3=\mathrm{B} 4,4=\mathrm{B} 3,5=\mathrm{B} 2,6=\mathrm{B} 1,7=\mathrm{A} 2$ e $8=A 1$. Então, o Qualis médio foi calculado como;

$$
\bar{x}_{j}=\sum_{i=0}^{8} i f r_{i j}
$$

Em que o qualis médio da área do conhecimento j é dado pelo somatório dos produtos entre posto e sua frequência relativa, ao longo de todos os possíveis Qualis.

Foi ajustado um modelo regressão linear múltipla considerando o fator de impacto como variável dependente e o JCR como variável independente para que pudesse ser traçada uma função que relacionasse as duas variáveis (Drapper; Smith, 1981).

A partir do estudo das variáveis supramencionadas foi possível traçar um perfil das métricas de avaliação de revistas o que contribui para autores e editores de revistas. Foram feitas as correlações entre as variáveis para verificar-se a existência de relação entre elas e assim iniciar-se as interpretações a partir do tratamento estatístico. O desenvolvimento do trabalho seguiu a ordem; coleta de dados, tratamento estatístico (cálculo de correlações entre as métricas), e por fim a interpretação dos resultados obtidos a partir da análise estatística.

\section{Resultados e discussão}

Em uma primeira análise, é possível constatar que as revistas que publicam na área de Ciências Agrárias I sofreram uma leve evolução qualitativa do triênio 2010-2012 para o quadriênio 2013-2016, que pode ser explicada pela atualização que os algoritmos de classificação sofreram nesse entremeio. Como pode-se observar na Figura 1, no primeiro período de avaliação $10 \%$ das revistas estavam classificadas no estrato A1 e no segundo período, este percentual subiu para $12 \%$. Aquelas avaliadas no estrato $C$ sofreram uma queda abrupta, saindo de $17 \%$ para apenas $1 \%$. Isto pode representar uma maior preocupação dos editores e autores em produzir conteúdos de maior qualidade e impacto na sociedade.

A situação representada na Figura 1 traz à tona a assimetria existente entre a proporção de periódicos que possuem Qualis A1 ou A2 versus os demais estratos. No triênio analisado, apenas $21 \%$ das revistas possuíam os dois estratos mais altos do Qualis, tal fato se repete no quadriênio, no qual apenas $23 \%$ eram identificados como A1 ou A2.

Talvez seja natural que o leitor se pergunte se aquelas revistas com maior Qualis tendem a melhores resultados em outros índices de avaliação. Ao decorrer deste artigo você leitor perceberá que isto nem sempre é uma regra.

Como foi supracitado a área de Ciências Agrárias I obteve um aumento relativo de qualidade, porém, é interessante saber quais são as outras áreas do conhecimento que tendem a ganhar Qualis a reboque, ou seja, quais são as áreas correlatas à área de Ciências Agrárias I e tendem a obter boas qualificações quando a área supramencionada também obtém. Para fazer esta investigação, foram construídos três gráficos, em que o primeiro traz informações a respeito da distribuição de frequência das áreas em que as revistas amostradas publicam (Figura 2) e os outros dois trazem uma comparação do Qualis médio das revistas nos dois intervalos de tempo estudados (Figura 3).

Os gráficos das Figuras 2 e 3 motivam discussões acerca da questão de que se as áreas do conhecimento em que as revistas publicam com maior frequência a despeito da área de Ciências Agrárias I tendem a obter qualificação no Qualis desta área. As barras em azul dessas figuras representam a área de Ciências Agrárias I. Assim como se esperava, ela é a maior, pois todas as revistas avaliadas têm ou já tiveram Qualis nesta área. As onze áreas subsequentes em que as revistas são mais bem qualificadas são, nessa ordem: Interdisciplinar, Biodiversidade, Biotecnologia, Ciências Ambientais, Ciências Biológicas I e II, Química, Zootecnia $\backslash$ Recursos Pesqueiros, Farmácia, Engenharias II e Medicina Veterinária. Destacaram-se 
onze áreas, pois desejava-se apontar para a posição da

que a área de Medicina Veterinária, uma vez que ela é uma área correlata à área de Ciências Agrárias I.

Figura 1 - Distribuição de frequências dos estratos do Qualis obtidos pelas revistas da área de ciências agrárias I

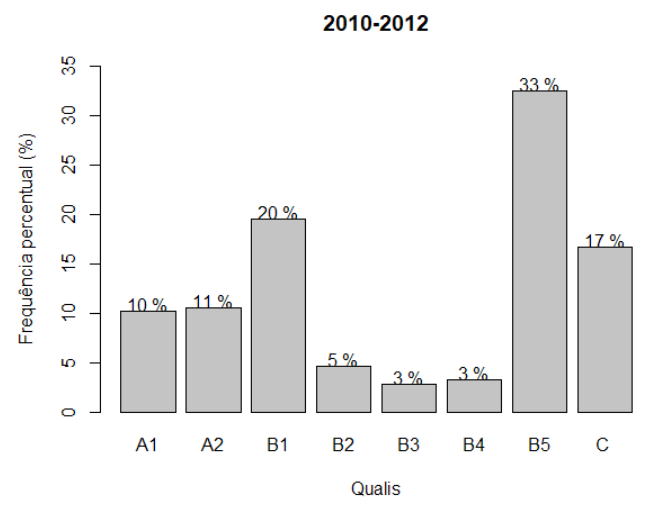

(A)

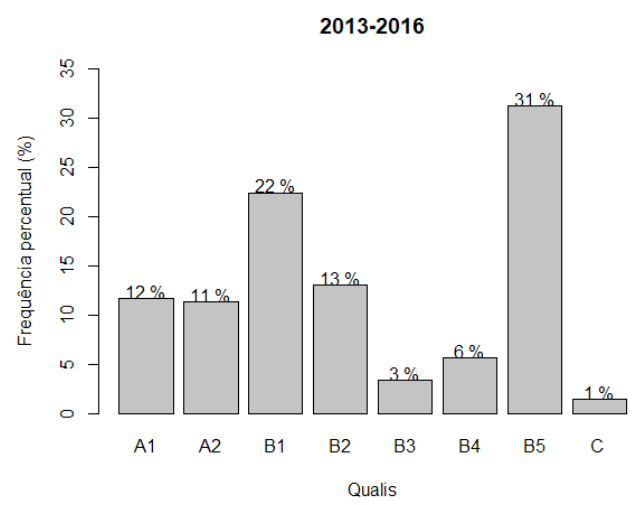

(B)

Distribuição de frequência no período 2010-2012. B- Distribuição de frequência no período 2013-2016.

Figura 2 - Distribuição de frequência percentual das áreas do conhecimento em que as revistas publicam

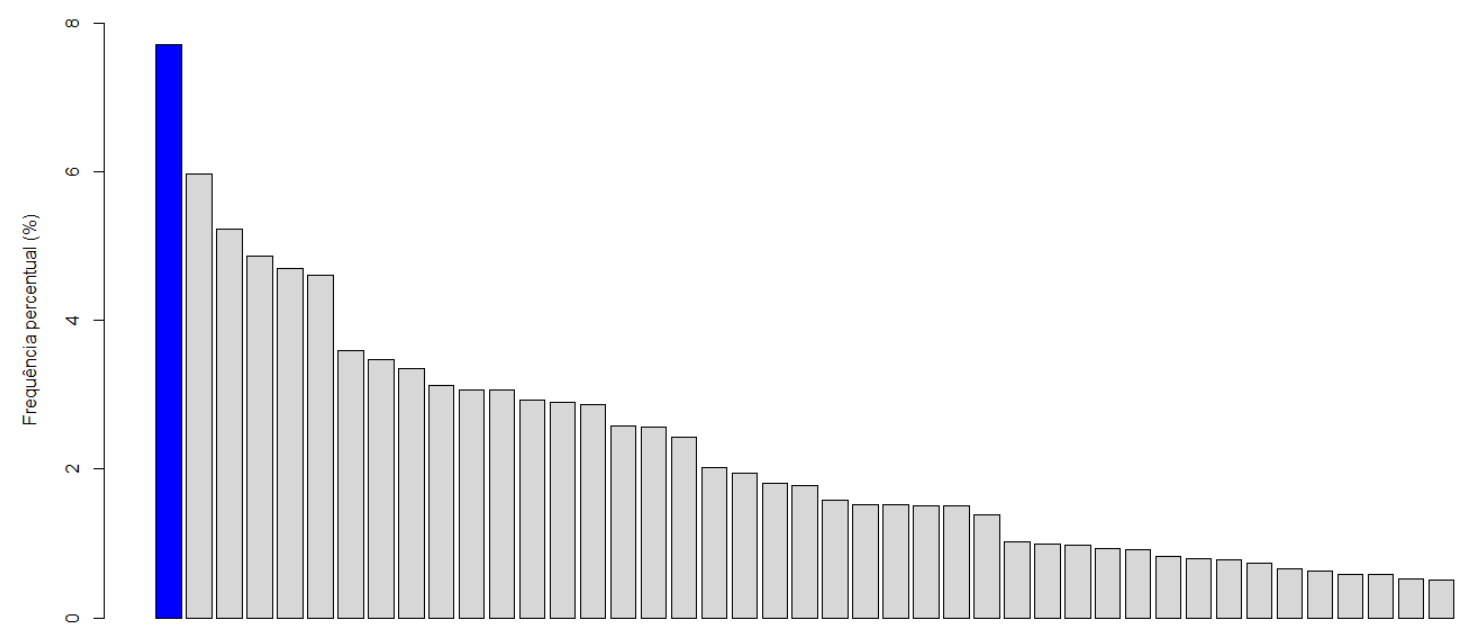

Áreas

Uma das mais plausíveis razões para a verificada qualificação conjunta nessas áreas é interseção existente entre os seus algoritmos de avaliação. Por exemplo, o JCR é fortemente utilizado para a determinação dos estratos superiores na área de Ciências Agrárias I, como pode ser visto pelo trecho extraído do relatório Qualis da área, com algumas adaptações de forma. De acordo com

[...] são identificados os periódicos nacionais e internacionais e os últimos foram identificados como "subject category" conforme a maneira que eles se encontram agrupados na base ISI Web of knowledge da Thomson Reuters (I) ou na base Scimago Journal \& Country Rank (S). [...] A plataforma Sucupira trouxe a "subject category" dos periódicos e foi utilizada a primeira indicada na plataforma como aquela preferencial do periódico. As revistas nacionais com JCR na base I e/ou CPD2 na base $\mathrm{S}$ de 5 anos maior que 1 e JCR e CPD2 (cites per doc dos últimos dois anos) maiores que 0,850 com publicações exclusivamente em inglês e forte aderência área forma classificadas como A1. Já aquelas com JCR na base I e/ou CPD2 na base S maiores ou iguais a 0,750 e menores que 1,00 e JCR e CPD2 de 2 anos maiores que 0,600 e menores que 0,850 com aderência a área e publicações exclusivamente em inglês foram classificadas como A2 [...] (Federizzi et al. 2016). 
O JCR também é critério de avaliação presente na área de Medicina Veterinária. De acordo com Miglino et al. (2017) "periódicos com JCR 3,029 foram qualificados em A1. Aqueles com 3,028 > JCR 2,041 foram qualificados em A2 e por fim, periódicos com 2,039 > JCR $\geq 0,729$ foram qualificados em B1 [...]".

Figura 3 - Qualis médio das revistas

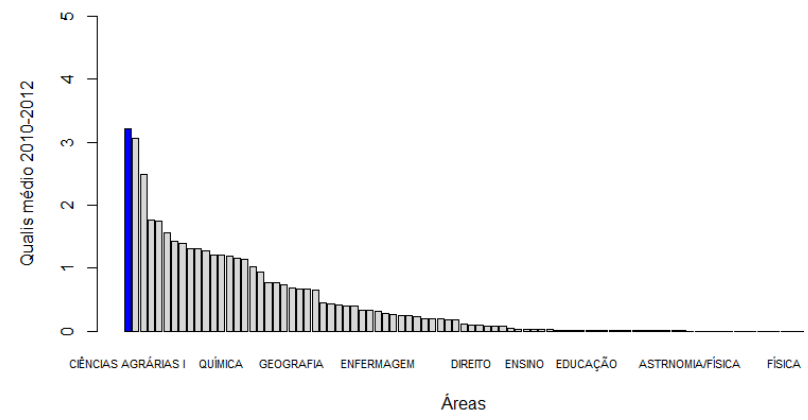

(A)
A partir da Figura 3 pode ser feita uma comparação entre a distribuição de frequência percentual (Figura 2) e o Qualis médio de cada área do conhecimento.

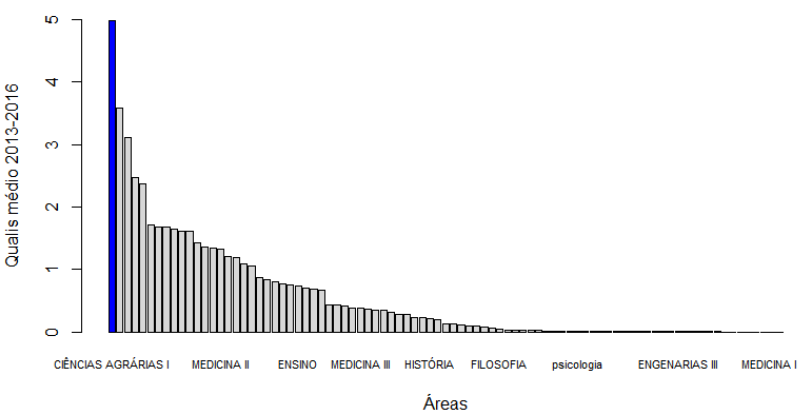

(B)

Qualis médio no período 2010-2012. B- Qualis médio no período 2013-2016.

Assim como na Figura 1, a barra em azul representa a área de Ciências Agrárias I. A partir de sua observação percebe-se que muitas das áreas que estão entre as maiores distribuições de frequência também possuem os maiores Qualis médios. Para fazer esta evidenciação, foram selecionadas as 11 áreas com os maiores Qualis médios de cada período de observação. O ranking segue na Tabela 1.

Tabela 1 - Qualis médio das áreas nos períodos 2010-2012 e 2013-2016

\section{Áreas com maior Qualis médio}

(2010-2012)
Áreas com maior Qualis médio (2013-2016)

\begin{tabular}{llll}
\hline Ciências Agrárias I & entre B4 e B3 & Ciências Agrárias I & entre B1 e B2 \\
Interdisciplinar & entre B4 e B3 & Interdisciplinar & entre B3 e B4 \\
Biodiversidade & entre B5 e B4 & Ciências Ambientais & entre B3 e B4 \\
Ciências Biológicas I & entre C e B5 & Biotecnologia & entre B5 e B4 \\
Biotecnologia & entre B4 e B3 & Biodiversidade & entre B5 e B4 \\
Ciências Ambientais & entre C e B5 & Ciências Biológicas I & entre C e B5 \\
Engenharias II & entre C e B5 & Engenharias II & entre C e B5 \\
Ciências Biológicas II & entre C e B5 & Zootecnia $\backslash$ Recursos Pesqueiros & entre C e B5 \\
Medicina II & entre C e B5 & Medicina Veterinária & entre C e B5 \\
Farmácia & entre C e B5 & Farmácia & entre C e B5 \\
Medicina Veterinária & entre C e B5 & Química & entre C e B5 \\
\hline
\end{tabular}

Além de ocupar a vice-liderança entre as frequências percentuais, a área Interdisciplinar repete o feito entre os Qualis médios, acompanhando a estratificação da área de Ciências Agrárias I de um período de avaliação para o outro, ou seja, enquanto o Qualis da área de Ciências Agrárias I sobe, o Qualis da área Interdisciplinar vem à reboque evidenciando a relação entre os algoritmos de avaliação destas áreas. Tal fato é observável pela similaridade entre os algoritmos de avaliação das duas áreas. Caminho similar é seguido pela área de Ciências Ambientais que também obteve um ganho qualitativo.

Porém, percebe-se que Biotecnologia se encontra entre as áreas mais publicadas mas, seu Qualis não seguiu o mesmo sentido da área de Ciências Agrárias I, obtendo um decréscimo de uma período para o outro. Além disto, a área de Engenharias II, que não está no ranking das 
mais publicadas, aparece no ranking dos maiores Qualis médios implicando que quando algum tema desta área é publicado ele tende a receber Qualis.

Outras variáveis, além do Qualis são estudadas neste trabalho, e deseja-se saber quais as correlações

Figura 4 - Correlação do Qualis com as demais métricas

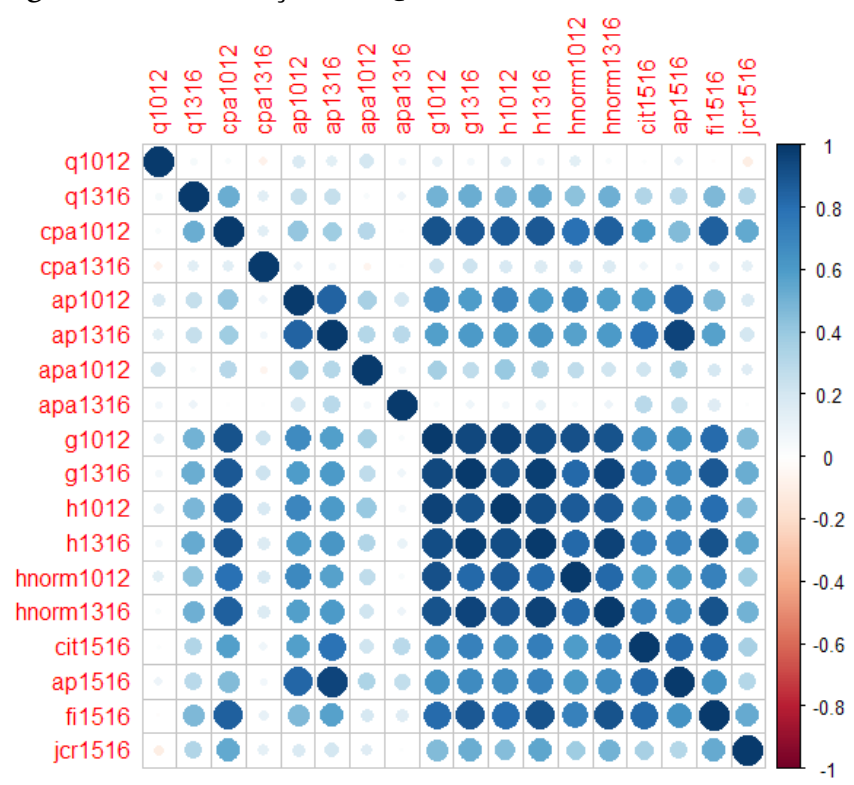

(A) existentes entre elas e o índice Qualis. Dentre estas variáveis destacam-se citações por artigo, artigos publicados, autores por artigo, índices $\mathrm{g}$ e h, índice h normalizado, fator de impacto (2015-2016) e JCR (2015-2016). Foram calculadas as correlações e os resultados foram colocados em uma matriz (Figura 4).

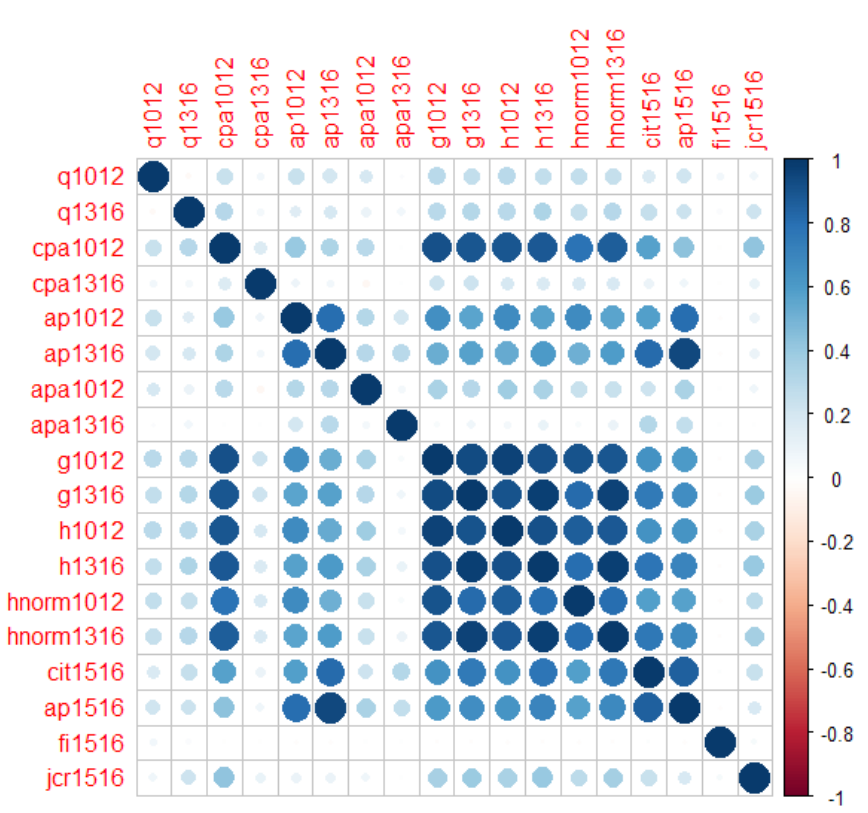

(B)

A - Correlação do Qualis da Área de Ciências Agrárias I com as demais métricas. B-Correlação do Qualis de todas as áreas do conhecimento com as demais métricas.

Em que : q: Qualis, cpa: citações por artigo, ap: artigos publicados, apa: autores por artigo, g:índice g, h: índice h, hinorm: índice h normalizado, cit: citações, fi: fator de impacto

Na Figura 4 são apresentadas duas figuras que representam as correlações entre as métricas estudadas neste trabalho. Nela, os círculos mais escuros representam as correlações mais fortes, enquanto os mais claros, as mais fracas. Além disso, a cor azul representa correlação positiva, branco representa correlação nula e vermelho, correlação negativa (não observada nesse estudo).

Na figura 4B percebe-se que a correlação entre o Qualis 2010-2012 e o Qualis 2013-2016 é inversa (-3,1\%), o que mostra uma mudança dos algoritmos e políticas de avaliação do Qualis de um período para o outro; tal fato se repete na Figura 4A.

Além disso, é possível notar que na Figura 4B existe uma baixa correlação entre o Qualis e os índices $h$ e g, implicando que para ambos os períodos estudados, ter Qualis alto não implica diretamente em obter boas pontuações nos índices internacionais de avaliação. Duas situações que corroboram para a conclusão acima são as das revistas, Academie des Sciences Comptes Rendus Biologies e Current Pharmaceutical Biotechnology. A primeira obteve no quadriênio 2013-2016 A2 em Ciências Agrárias I, índices $\mathrm{g}$ e h, 30 e 24, respectivamente. A segunda, neste mesmo período, obteve Qualis B1, índices g e h, 34 e 25 respectivamente. De acordo com Oliveira et al. (2015), esta situação revela que os critérios utilizados para a avaliação nacional não são quantitativos ou não consideram o número de citações dos artigos; o que é confirmado pelo fato da correlação entre o Qualis e citações por artigo ser tão baixa. Além disto, conclui-se até aqui que existe um desalinhamento entre o Qualis e os demais índices de avaliação.

Já na Figura 4A o cenário tem uma sutil mudança. Os índices h, g e h normalizado, possuem correlação mais forte especialmente com o Qualis 2013-2016, dando à entender que as mudanças da política de avaliação do Qualis do biênio (2010-2012) para o triênio (2013-2016) afetaram mais diretamente a área de Ciências Agrárias I em detrimento das demais. Porém ainda é evidente que a métrica qualitativa segue caminhos distintos dos traçados pelas quantitativas.

Outro ponto de discussão que é instigada pela Figura 4 B e que o leitor deve se interessar, é a pequena 
correlação entre o Qualis 2013-2016 e o JCR correspondente a este período (JCR 2015-2016), apenas 23\%, o que é no mínimo curioso já que vários algoritmos de avaliação do Qualis utilizam o fator de impacto JCR como um critério de qualificação. Tal fato tende a se repetir na Figura 4A, porém, com uma correlação um pouco mais forte $(32 \%)$.

Na Figura 4A e B evidencia-se a alta correlação entre o número de citações por artigo no triênio com os índices $\mathrm{g}$, h e hi ${ }_{\text {norm }}$ estudados em ambos os períodos; o que era esperado, já que os índices citados são calculados a partir do número de citações do artigo. Além disso, o número de citações de 2015-2016 também é influenciado por esta métrica. O número de artigos publicados tanto para o triênio quanto para o quadriênio tem correlação direta com todos os índices para ambos os períodos; isto indica que quanto mais artigos uma revista publica maior a chance de obter boas qualificações em outras métricas de avaliação.

A partir das discussões feitas até então, pode-se concluir que autores que publicam em revistas com Qualis alto esperando grande impacto, devem estar atentos pois, de maneira geral o Qualis possui baixa correlação com as métricas quantitativas que avaliam a qualidade da produção científica.

O presente trabalho não tem por intuito focar exclusivamente nas questões que tangenciam o índice Qualis, mas também fazer análises acerca do fator de impacto (FI) e sua versão paga o JCR. Em tese, estas duas métricas possuem grande similaridade porém, de acordo com o estudo realizado para a construção deste trabalho isto nem sempre ocorre.

Para tanto, foi feita uma comparação entre as variáveis supracitadas com o intuito de inferir sobre as correlações entre elas. Para isto, foi utilizado o coeficiente de correlação de Spearman, ideal para o estudo de variáveis contínuas tais quais estas, no qual obteve-se uma correlação de 53\%, indicando uma correlação mediana entre as variáveis. Porém, esta correlação gera grande estranheza já que o cálculo utilizado para a obtenção das duas métricas é o mesmo. Logo, esperava-se uma correlação mais alta entre estes dois índices. Essa discrepância pode advir do fato do JCR ser calculado utilizando-se das informações das revistas que estão contidas nas bases do ISI. Outra explicação plausível é dada por Pinto e Andrade (1999), no qual os autores trazem em seu trabalho discussões sobre o fato de existirem alguns dados incorretos na base SCI. De acordo com eles, erros como; nome do autor errado, nome da revista errado e autores citados fora de ordem.

Dessa forma, revistas que não possuem orçamento contratarem o serviço de monitoramento pela Clarivate Analitycs ${ }^{\circledR}$ não conhecem seu impacto via JCR. Alternativamente, podem estimar seu impacto a partir de plataformas gratuitas como o Google Acadêmico, por exemplo. A questão a ser discutida éque índices de avaliação de programas de pós-graduação como o Qualis, utilizam o JCR como forte critério para a qualificação de revistas (como na área de Ciências Agrárias I). Logo, aquelas que não o possuem tendem a receber Qualis menor, já que este fato culmina na não atratividade de autores e trabalhos melhores.

Packer (2010) propõe que "o índice Qualis enriqueça sua avaliação com uso de múltiplas medidas de desempenho". Isto ataca justamente a questão do Qualis dar grande importância e relevância para o fator de impacto JCR, desconsiderando outras formas de medir o impacto das revistas.

Diante deste fato é plausível a discussão do que é considerada ciência pela Capes. Pelo que parece, a produção de científica só possui qualidade se for possível pagar por ela.

A partir destas discussões, foi construído um modelo de regressão que pudesse relacionar de forma mais clara o JCR e o fator de impacto (Figura 5).

Figura 5 - Modelos de regressão do JCR em função do fator de impacto

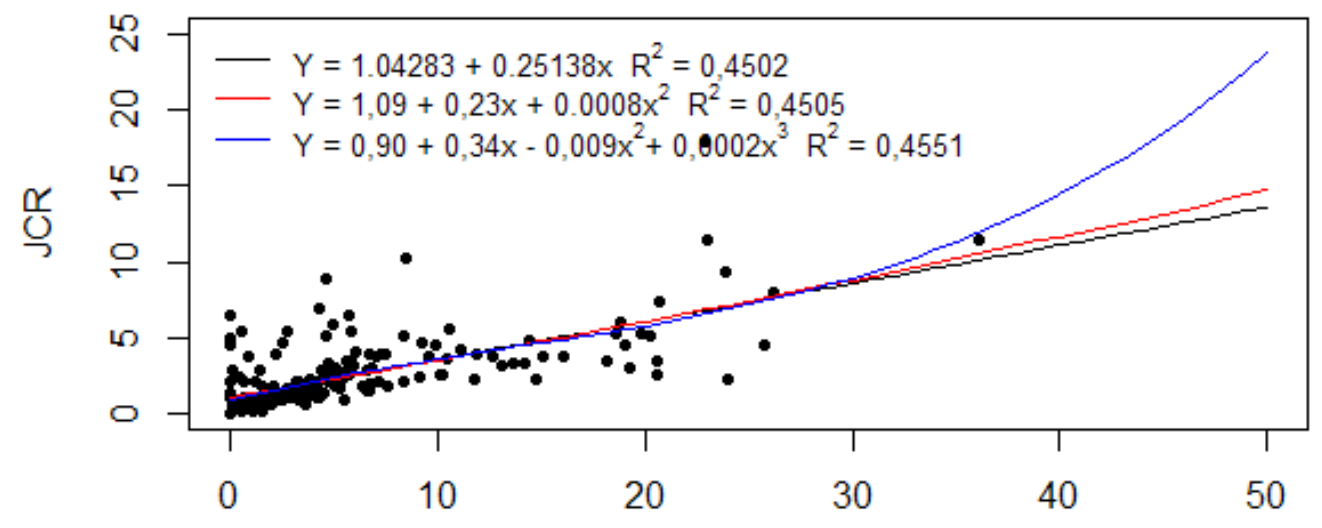

Fator de impacto

Modelo de grau 1 (preto), de grau 2 (vermelho) e de grau 3 (azul) 
Gonçalves Junior, E. M.; Ferreira, E. B.

A nuvem de pontos da Figura contém os fatores de impacto baseados no Google Acadêmico e os JCR cada de umas das revistas analisadas neste trabalho. A partir desses pontos foram ajustados 3 modelos de regressão com intuito de descobrir aquele que melhor explicasse a relação entre as variáveis estudadas. O modelo linear explicou $45,02 \%$ da relação, enquanto os modelos quadrático e cúbico explicaram 45,05\% e 45,51\% respectivamente.

Devido a pequena diferença entre a variância de modelos encaixados, o teste de razão de verossimilhanças mostrou não haver vantagem em utilizar os modelos de segundo e terceiro grau e, por parcimônia, elegeu-se a reta como melhor modelo. Diante deste fato, pode-se utilizar a função linear, , em que y é o JCR e x é o fator de impacto do Google Acadêmico, para obter o JCR a partir do fator de impacto. Este fato é de grande importância, pois autores e periódicos podem fazer o cálculo aproximado de uma métrica paga, a partir de uma métrica gratuita.

\section{Conclusão}

O desenvolvimento do presente trabalho possibilitou uma análise mais profunda acerca dos algoritmos de avaliação que regem a produção científica brasileira, evidenciando relações entre as métricas e descobrindo vieses entre elas. Com isso foi possível cumprir com os objetivos traçados no início da pesquisa. Com os resultados apresentados no corpo deste trabalho, acredita-se que pesquisadores, autores e editores de revistas usufruam do conhecimento científico produzido para gerar uma ciência que se adapte cada vez mais as normas de avaliação vigentes.
O presente trabalho produziu como principais resultados uma correlação mediana entre o fator de impacto geral e o JCR, sendo esta igual a 53\%, o que não era esperado, já que se imaginava uma correlação forte entre as métricas. Encontrou-se por meio do método de regressão linear uma função que permite calcular o JCR por meio do fator de impacto, sendo esta JCR $=1.04283$ + 0.25138·fi. Evidenciou-se também que o índice Qualis (no geral) não é determinante para se obter JCR, citações, índice $\mathrm{h}$, índice $\mathrm{g}$, já que as correlações são baixas, ou seja, ter um bom Qualis não implica ter impacto. Também se evidenciou que em 2010-2012, 10\% das revistas possuíam Qualis A1 e que em 2013-2016 12\% delas estavam classificadas neste estrato.

Outra observação interessante foi a queda abrupta do Qualis C do primeiro para o segundo período, saindo de $17 \%$ para apenas 1\%. Existem diversos materiais que discutem sobre cienciometria porém, materiais que discutem sobre as relações entre as métricas são escassos, culminando em uma dificuldade para a discussão dos resultados.

Alguns materiais encontrados confirmaram a baixa correlação entre os índices h e g e o Qualis. Com a execução deste trabalho foi possível obter-se um grandioso acumulo de conhecimento acerca das métricas de avaliação de revistas e suas relações. Além disto, sugere-se uma nova visão em relação à avaliação da ciência produzida no Brasil dando-se mais incentivo aos periódicos nacionais para que se possa ter um melhor desempenho internacionalmente. Espera-se que novos trabalhos como este possam surgir, com novas perspectivas e resultados importantes para a ciência nacional.

\section{Referências}

Barata, R. C. B. 2016. Dez coisas que você deveria saber sobre o Qualis. Revista Brasileira de Pós-graduação, 13: 13-40. DOI: 10.21713/23582332.2016.v13.947.

Drapper, N. R; Smith, H. 1981. Applied Regression Analysis. 2. ed. New York. John Wiley \& Sons.

Egghe, L. 2006. Theory and practise of the g-index. Scientometrics, 69: 131-152. Disponível em: https://bit.ly/2oJxlWo.

Federizzi, L. C; Vitorino, A. C. T; Lopes, S; Lopes, M. T. G; Chagas, E. A. 2016. Critérios de classificação Qualis: Ciências Agrárias I. Disponível em: https://goo.gl/C9s6S3.

Ferreira, A. G. C.. 2010. Bibliometria na avaliação de periódicos científicos. Revista em Ciência da Informação, 11. Disponível em: https://bit.ly/2lwx7Qq.

Harzing, A.W. Publish or Perish. 2007. Disponível em: http://www. harzing.com/pop.html.

Harzing, A.W; Alakangas, S.; Adams, D. 2014. HIa: An individual annual h-index to accommodate disciplinary and career length differences, Scientometrics, 99: 811-821. DOI: 1007/s11192-013-1208-0.
Hirsch, J. E. 2005. An index to quantify an individual's scientific research output. Proceedings of the National Academy of Sciences of the United States of America (PNAS), 102:16569-16572. DOI: https:// doi.org/10.1073/pnas.0507655102

Hayashi, M.C.P. 2012. Sociologia da ciência, bibliometria e cientometria: contribuições para a análise da produção científica. Anais do Seminário de Epistemologia e Teorias de Educação (EPISTED), Campinas, SP, Brasil, 4. Dísponível em: https://bit.ly/21AiBqM.

Oliveira, A. B.; Rodrigues, R. S.; Blattmann, U.; Pinto, A. L. 2015. Comparação entre o qualis/capes e os índices h e g: o caso do portal de periódicos UFSC. Informação e Informação, 20: 70-91. DOI: https:// bit.ly/2lzRdJO.

Packer, A. 2010. Os Periódicos Brasileiros e a Comunicação da Pesquisa Nacional. Revista USP, 20: 26-61. Disponível em: https://bit.ly/2lPZRDQ.

Pinto, A. C. ; Andrade, J. D. 1999. Fator de Impacto de Revistas Científicas: qual o Significado deste Parâmetro? Química Nova, 22: 448-453. DOI: https://bit.ly/2xqqXoT.

Siegel, S.; Castellan Junior, N. J. 2006. Estatística não paramétrica para as ciências do comportamento. p. 266-276. In: Métodos de associação e seus testes de significância. Bookman, São Paulo. Artmed. 